Cernadas Ríos, Francisco X.; Santos Rego, Miguel A.; Lorenzo Moledo, María del Mar (2013). Los profesores āntē la educación intercultural: el desafío de la formación sobre el terreno. Revista de Investigación Educativa, 31 (2), 555-570.

http://dx.doi.org/10.6018/rie.31.2.155391

\title{
LOS PROFESORES ANTE LA EDUCACIÓN INTERCULTURAL: EL DESAFÍO DE LA FORMACIÓN SOBRE ELTERRENO
}

\author{
Francisco X. Cernadas Ríos ${ }^{(1)}$, Miguel A. Santos Rego ${ }^{(2)}$ y María del Mar Lorenzo Moledo(2) \\ (1) CEIP A Maía (A Coruña) \\ (2) Universidad de Santiago de Compostela
}

\section{RESUMEN}

La pluralidad que supone la presencia en las aulas del alumnado inmigrante reclama una respuesta apropiada por parte del sistema educativo. En este artículo se analiza la percepción del profesorado de Educación Infantil y Primaria sobre sus necesidades de formación en Educación Intercultural. Se trata de un estudio descriptivo de encuesta con una muestra de 368 docentes de Educación Infantil y Primaria de Galicia. Los resultados mostraron que este colectivo ve necesario incluir la Educación Intercultural en la formación del profesorado. También se constató que la edad y la experiencia profesional influyeron significativamente en las valoraciones de los docentes. La etapa en la que se imparte docencia, la titulación académica y la interacción con el alumnado inmigrante no tuvieron una valoración significativa en la opinión del colectivo docente.

Palabras clave: Formación del profesorado; educación intercultural; percepción de los profesores; desafios de futuro.

\footnotetext{
Correspondencia:

Francisco X. Cernadas Ríos, CEIP A Maía, s/n. 15.220 Bertamirán-Ames (A Coruña). Telf. 981883152. Fax. 9818902 34. E-mail: fucocernadas@edu.xunta.es

Miguel A. Santos Rego, Facultad de Ciencias de la Educación. Campus Vida. Universidad de Santiago de Compostela. 15782 Santiago de Compostela. Telf. 881813755. E-mail: miguelangel.santos@usc.es

María del Mar Lorenzo Moledo, Facultad de Ciencias de la Educación. Campus Vida. Universidad de Santiago de Compostela. 15782 Santiago de Compostela. Telf. 881813758. E-mail: mdelmar.lorenzo@usc.es
} 


\title{
TEACHERS FACING INTERCULTURAL EDUCATION: THE CHALLENGE OF IN SITUTRAINING PROGRAMS
}

\begin{abstract}
The diversity of students in today's classrooms because of immigration demands a suitable response on behalf of education systems. This study analyzed Pre-school and Primary Education teachers' perception of their training needs for Intercultural Education using a descriptive survey design. The sample comprised 368 Pre-school and Primary Education teachers in Galicia, Spain. The results showed that participants regarded as necessary the introduction of Intercultural Education in teacher training. Age and professional experience played a significant role in teachers' opinions, while school year, teachers' qualifications, and interaction with immigrant students did not have an impact on teachers' opinion.
\end{abstract}

Keywords: Teacher training; intercultural education; teachers' perception; future challenges.

\section{INTRODUCCIÓN}

La diversidad cultural, presente históricamente en España en razón fundamentalmente de la existencia de diferentes comunidades nacionales y también de la minoría gitana, se vio incrementada posteriormente en virtud del proceso de globalización y las migraciones a las que ésta ha dado origen. En Galicia, el colectivo de escolares de procedencia inmigrante, originarios en su mayoría de Centro y Sudamérica, representa en la actualidad un 3,5\% del total (MEC, 2011). A esta diversificación del alumnado, que barre definitivamente el carácter unificador de la actividad escolar en el ámbito de la cultura, la lengua y las costumbres, ha venido a unirse además el cambio desde una concepción de la educación como privilegio reservado a los mejores, hasta su consideración como un derecho, como un servicio público que debe ofrecerse, sin exclusiones, también a los niños con más dificultades para seguir el ritmo de la enseñanza (Esteve, 2004, p. 95).

La pluralidad que supone la presencia del alumnado inmigrante reclama una respuesta apropiada por parte del sistema educativo, al que corresponde asumir un rol protagonista a la hora de resolver el denso rompecabezas de variables que nos han de permitir una convivencia fundamentada en la equidad y la igualdad de derechos para todos (Essomba, 2006, p. 8). La existencia de estas minorías, lejos de suponer un problema, habrá de contemplarse como una buena oportunidad para educar a los sujetos en valores democráticos como la tolerancia, el diálogo, la solidaridad, la resolución de conflictos y el sentimiento de pertenencia al grupo (García Llamas, 2005, p. 107). La Educación Intercultural (EI) se presenta en este contexto como el enfoque pedagógico más apropiado, constituyendo además la orientación imprescindible para la supervivencia y actualización de las instituciones educativas.

En la práctica, sin embargo, pese al interés y la preocupación que ha provocado esta cuestión, y por más que se insista en el papel fundamental de la escuela como vector de integración social y cultural de los hijos de emigrantes y de sus familias, las estrategias puestas en marcha a fin de facilitar la incorporación del alumnado extranjero a 
las escuelas siguen sin alcanzar los resultados deseados (Santos, Lorenzo \& Priegue, 2011, p. 98). El tratamiento pedagógico que se viene dando a la diversidad cultural se asimila al propio de una circunstancia excepcional o anormal y se resuelve con el establecimiento de algunas medidas especiales compensatorias dirigidas a ciertos grupos diana. Y es que el fenómeno migratorio ha puesto en evidencia la escasa cualificación de los profesionales de la educación al respecto (Santos \& Lorenzo, 2003, p. 68). Hasta ahora, el profesorado no está recibiendo en su formación inicial, ni tampoco dentro de las distintas modalidades de formación permanente, una preparación específica para elaborar verdaderos currículos interculturales (Marcelo, 1992; Peñalva \& Soriano, 2010). Además, las iniciativas de formación en este campo se preocupan básicamente por elevar el nivel de sensibilización únicamente de aquellos docentes que están en contacto directo con los alumnos minoritarios. Por otra parte, el modelo de formación en el que el profesor actúa como un mero consumidor de formulaciones ajenas, además de haberse revelado incapaz de producir los necesarios cambios de valores y actitudes en los docentes, trae como consecuencia una inadecuada conceptualización de la EI, que es vista entre una buena parte de los mismos como algo que no modifica su práctica educativa, afecta solamente a la materia de ciencias sociales y puede incorporarse como una lección más, al margen del currículo (Díaz-Aguado, 2003).

Diversos informes (Colectivo IOÉ, 1995; Defensor del Pueblo, 2003) destacan la actitud claramente favorable del profesorado ante la presencia del alumnado inmigrante, si bien es igualmente cierto que a menudo se produce una discontinuidad o incoherencia entre lo que los educadores suelen manifestar en los cuestionarios formales y su íntima consideración de esta presencia como una situación problemática e incómoda (Garreta \& Llevot, 2003). Entre los profesionales que trabajan con inmigrantes, a pesar de que en general conocen el sentido de la interculturalidad, son mayoría quienes confiesan no poder traducir en la práctica lo que supondría la misma, al no disponer de suficientes recursos formativos (Escarbajal, 2010, p.168). Con el objetivo de alcanzar un correcto desarrollo de la EI, resulta indispensable que el profesorado adquiera las denominadas "competencias interculturales", es decir, las habilidades cognitivas, afectivas y comunicativas necesarias para manejarse eficazmente en un medio intercultural (ver McAllister \& Jordan, 2000; Sercu, 2006; Leiva, 2010). Las dimensiones principales de un programa de formación en este campo podrían ser, por lo tanto, las siguientes (Jordán, 2004): la dimensión cognitiva o teórica, en la que los elementos de carácter informativo deberán ir seguidos del examen crítico sobre una serie de temas que permitan comprender la complejidad del hecho multicultural, la dimensión técnico-pedagógica (proporcionando estrategias organizativo-didácticas) y, por último -y aún de forma prioritaria-, la dimensión emocional-actitudinal-moral, es decir, el necesario cambio de actitudes, el aspecto más delicado y relevante, y también el más indigente hoy por hoy (Jordán, 1994). Consecuentemente, no basta con incluir asignaturas obligatorias sobre EI, diversidad cultural, etc. en los planes de estudio; se hace necesario (Nieto \& Santos, 1997) transformar totalmente los programas de formación del profesorado para reelaborarlos desde un enfoque intercultural (cfr. Aguado, Gil \& Mata, 2008; Grupo Inter, 2006).

Como superación del modelo de racionalidad técnica, considerando el contexto como realidad socialmente construida, surge en el ámbito de la formación del profesorado el 
modelo de "reflexión en la acción" (Schön, 1998) o "enfoque reflexivo sobre la práctica" (Pérez Gómez, 1992). Desde esta perspectiva teórica, el profesorado es visto como un agente de cambio y un crítico comprometido (Giroux, 1997), esto es, como un profesional reflexivo que trabaja en equipo siguiendo el método de investigación-acción (Elliot, 1997), capaz de analizar los mecanismos de control social del conocimiento, el poder de las estructuras y las instituciones sociales, y competente para desarrollar destrezas que cuentan en la acción social. Entre las características más relevantes de este modelo de formación, López Reillo (2006, p. 129) destaca las siguientes: colaboración con el resto del profesorado, interés por el contexto inmediato de la práctica educativa y sus conexiones con el contexto mediato, capacidad para afrontar situaciones problemáticas prácticas, producción de conocimiento y comprensión del mismo en la acción y estrecha relación entre formación y desarrollo profesional que concede al Centro Educativo el protagonismo absoluto en cuanto a lugar de acción.

En este trabajo, se analizan los resultados de una encuesta realizada entre el profesorado gallego con el propósito de aproximarnos al examen de las percepciones docentes en relación con su propia formación en EI, las necesidades de preparación sentidas y sobre cuales pudieran ser las modalidades y contenidos de formación más apropiados en este campo.

Este estudio se plantea como objetivo general indagar sobre la formación en EI del profesorado de Galicia. Este objetivo se concreta en tres objetivos específicos:

1. Evaluar el nivel de formación del colectivo docente en diversidad cultural.

2. Analizar la opinión del profesorado en torno al tipo de formación necesaria para la atención a la diversidad cultural en la escuela.

3. Analizar la influencia de las variables edad, nivel de formación específica en diversidad cultural, situación profesional y género en la formación del colectivo docente.

\section{METODOLOGíA DE LA INVESTIGACIÓN}

\section{Diseño}

Entre las diferentes estrategias de análisis comúnmente utilizadas en la investigación social y educativa, optamos por una metodología fundamentalmente cuantitativa, no experimental, de tipo ex-post-facto, con el propósito de intentar describir la realidad existente. Concretamente, se trata de un estudio de encuesta, en el que, como instrumento para la recogida de información, diseñamos un cuestionario en el que la mayor parte de los ítems son preguntas cerradas, redactadas en forma de escala Likert.

\section{Participantes}

La población o universo al que referimos nuestra indagación es el profesorado de Educación Infantil y Primaria de la enseñanza pública en la Comunidad Autónoma de Galicia. Para la selección de la muestra, recurrimos a la técnica de muestreo por conglomerados o conjuntos (Fox, 1981), utilizando los colegios como unidad muestral y escogiendo centros urbanos que contasen con una presencia importante de alumnado 
extranjero. La muestra final productora de datos quedó conformada por 368 docentes de 40 Centros públicos ${ }^{1}$ de Educación Infantil y/o de Educación Primaria de prácticamente todas las grandes ciudades pertenecientes a las cuatro provincias gallegas.

El perfil del profesorado que respondió a la encuesta puede sintetizarse en las siguientes características personales y profesionales: 281 docentes eran mujeres y 87 hombres, con edades que superaban, mayoritariamente, los 41 años (el 29,9\% tenía entre 41 y 50 años; el 42,1\% superaba esta edad). El 78,7\% de ellos contaba con plaza en Propiedad Definitiva. Se trataba fundamentalmente de diplomados (68,9\%), aunque el $30,8 \%$ tenían la titulación de licenciatura. Tenían una dilatada experiencia docente, ya que el 71,4\% acumulaba más de 16 años de servicio. Por último, el 59,4\% de la muestra llevaba más de 3 años trabajando en programas de atención al alumnado inmigrante.

\section{Instrumentos y procedimiento}

Como instrumento para la recogida de datos, elegimos la aplicación de la técnica más utilizada en la investigación por encuesta, un cuestionario confeccionado expresamente para este estudio a partir de una extensa revisión bibliográfica sobre el tema.

El instrumento de medida utilizado reúne los dos requisitos esenciales de fiabilidad y validez. Para evaluar el nivel de fiabilidad, o exactitud de las medidas, utilizamos el método de consistencia interna denominado Coeficiente alfa de Cronbach. Una vez aplicado este coeficiente a los resultados de nuestra escala, obtuvimos para la totalidad de las variables un valor numérico de 0,716 (Alfa de Cronbach basada en los elementos tipificados: 0,721. № de elementos: 61), lo que garantiza el cumplimiento de este criterio.

La validez del cuestionario viene garantizada por la realización, previa a su aplicación generalizada, de una prueba piloto a un grupo de diez docentes, con el propósito de detectar posibles fallos o errores de comprensión e interpretación y medir el tiempo de cumplimentación, efectuando posteriormente las oportunas rectificaciones y mejoras de algunos ítems.

Las encuestas fueron entregadas en mano a alrededor de 950 profesores en sus propios centros de trabajo, tras haberles proporcionado información sobre el objetivo de la investigación y normas de cumplimentación de los impresos. Se garantizó la confidencialidad de los datos obtenidos y su uso exclusivo en el marco de la propia investigación, mostrando además el agradecimiento a los participantes por su colaboración.

\section{Codificación de los datos}

Una vez codificadas las respuestas a los diferentes ítems del cuestionario, toda la información fue introducida en una matriz de datos utilizando el paquete estadístico Statistical Package for the Social Science (SPSS). Realizamos a continuación un estudio estadístico descriptivo de cada una de las variables por separado, y posteriormente comparativo en función de determinadas variables. Para determinar la significación estadística de las diferencias encontradas en función de algunas características per-

1 Se incluyen en este cómputo únicamente aquellos establecimientos educativos representados en la muestra por al menos cuatro docentes que hubiesen contestado el cuestionario. 
sonales y profesionales, empleamos la prueba $t$ de Student en el caso de las variables dicotómicas y el Análisis de Varianza (ANOVA) para el resto, utilizando el Método Sheffé en el análisis dirigido a establecer entre que grupos se producían las diferencias.

\section{RESULTADOSY DISCUSIÓN}

El objetivo central de esta investigación se centró en la formación en EI del profesorado de Galicia.

\section{Nivel de formación y opinión del colectivo docente en diversidad cultural}

El grado de implicación del profesorado en programas de preparación docente para trabajar con alumnado culturalmente diverso resultó escasamente alentador: apenas un tercio del profesorado $(29,8 \%)$ había participado a lo largo de su vida profesional en alguna de las actividades de formación específica ofertadas, organizadas en su mayoría por los Centros de Formación e Recursos (CEFORE).

Entre las motivaciones fundamentales que los alientan a participar en procesos formativos, los encuestados destacaron como estímulo fundamental el perfeccionamiento en métodos y técnicas de enseñanza $(85,2 \%)$ y, en segundo lugar, el aprendizaje del manejo de las Tecnologías de la Información y la Comunicación (55,3\%). Se observó un escaso interés declarado $(13,7 \%)$ por la simple acumulación administrativa de horas de formación para acreditar sexenios ${ }^{2}$.

También se les pidió a los docentes de este estudio que anotasen el título y año de celebración de las dos últimas actividades de formación (jornadas, cursos...) a las que hubiesen asistido. Los datos reflejaron la presencia de un elevado porcentaje de actividades relacionadas con las Tecnologías de la Información y la Comunicación (32\%), junto al escaso número de las que eran susceptibles de ser conectadas con la inmigración y la interculturalidad (7\%). Se observó que la mitad de estas actividades tuvo lugar en el año inmediatamente anterior (2007), lo que revela un muy aceptable nivel de asiduidad en la participación del profesorado en procesos formativos.

Sorprende que, a pesar de la baja participación en actividades formativas en EI, tan solo la mitad $(45,5 \%)$ del profesorado reconoció no tener suficiente formación en este ámbito. En cuanto al nivel genérico de confianza en la preparación de los profesores/ as para responder a los desafíos de la EI, únicamente una de cada cinco personas de la muestra (21,2\%) confiaba en la idoneidad de la misma, ascendiendo al 59,5\% el porcentaje de quienes estaban convencidos de la insuficiencia del conocimiento en este campo. Cabe destacar que la valoración concedida por el profesorado a la formación de sus colegas resultaba inferior a la otorgada a la preparación propia, tal como puede verificarse contrastando las respectivas medias: 3,43 en este ítem frente al 3,20 en el anterior.

2 Es preciso señalar que la Administración Educativa supedita el reconocimiento del complemento salarial denominado sexenios a la acreditación por el profesor/a de su participación, a lo largo de los seis últimos años, en un mínimo de 100 horas de actividades formativas, otorgando validez -para tales efectosúnicamente a las organizadas por determinadas instituciones, particularmente los CEFORE y los sindicatos de la enseñanza. 
Como parte del proceso de mejora de la enseñanza de los niños/as de procedencia inmigrante, cuatro de cada cinco docentes encuestados $(79,2 \%)$ reconocieron la importancia de la intervención de otros agentes externos a la escuela. En esta misma línea, la conveniencia de que los centros con un número notable de alumnos/as inmigrantes cuenten con mediadores/as interculturales fue favorablemente considerada por el 84,7\% de los profesionales.

La mayoría del profesorado (83,4\%) era consciente de la necesidad de incluir la EI en la formación inicial de los docentes. Además, consideraba inadecuado (61\%) el nivel de la atención dispensada a este tema en la formación continuada, llamando particularmente la atención el elevado porcentaje $(24,5 \%)$ de quienes se mostraban indiferentes.

TABLA 1

PERCEPCIONES DOCENTES RELATIVAS A LA FORMACIÓN EN EI

\begin{tabular}{|c|c|c|c|c|c|c|c|}
\hline & \multicolumn{2}{|c|}{ Conformidad } & \multirow[b]{2}{*}{ 莺 } & \multicolumn{2}{|c|}{ Disconformidad } & \multirow[b]{2}{*}{$\stackrel{\pi}{\stackrel{\tilde{\Xi}}{\Sigma}}$} & \multirow[b]{2}{*}{ 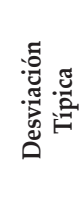 } \\
\hline & 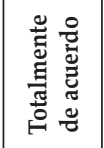 & 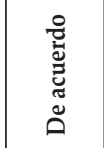 & & 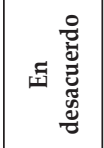 & 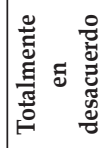 & & \\
\hline $\begin{array}{l}\text { Considero que mi formación en Educación } \\
\text { Intercultural es adecuada }\end{array}$ & $3,6 \%$ & $25,4 \%$ & $25,4 \%$ & $38,8 \%$ & $6,7 \%$ & 3,20 & 1,010 \\
\hline $\begin{array}{l}\text { Los profesores/as, en general, estamos pre- } \\
\text { parados para responder a los desafíos de la } \\
\text { Educación Intercultural }\end{array}$ & $2,5 \%$ & $18,7 \%$ & $19,3 \%$ & $52,6 \%$ & $6,9 \%$ & 3,43 & ,953 \\
\hline $\begin{array}{l}\text { La intervención de otros agentes externos } \\
\text { a la escuela es importante para mejorar la } \\
\text { enseñanza de los niños/as inmigrantes }\end{array}$ & $9,4 \%$ & $59,8 \%$ & $12,5 \%$ & $6,1 \%$ & $2,2 \%$ & 2,12 & ,866 \\
\hline $\begin{array}{l}\text { Sería positivo que en centros con un núme- } \\
\text { ro notable de alumnos/as inmigrantes hu- } \\
\text { biera mediadores/as interculturales }\end{array}$ & $26,5 \%$ & $58,2 \%$ & $9,7 \%$ & $3,1 \%$ & $2,5 \%$ & 1,97 & 843 \\
\hline $\begin{array}{l}\text { La formación inicial del profesorado tiene } \\
\text { que incluir Educación Intercultural }\end{array}$ & $31,5 \%$ & $51,9 \%$ & $10,8 \%$ & 3 & $2,8 \%$ & 1,94 & 889 \\
\hline $\begin{array}{l}\text { En la formación continuada del profesora- } \\
\text { do este tema está suficientemente atendido }\end{array}$ & $2 \%$ & $12,5 \%$ & $24,5 \%$ & $49 \%$ & $12 \%$ & 3,56 & ,92 \\
\hline
\end{tabular}

Entre las diferentes modalidades de preparación específica del profesorado en este ámbito, la más escogida hacía referencia al conocimiento de otras experiencias exitosas, a fin de adaptarlas al propio contexto (91,4\%). Esto se debería, según Jordán (1994, pp.145-146), al predominio de una mentalidad practicista en el profesorado, que se traducía, sobre todo, en la búsqueda de procedimientos muy concretos y funcionales. Le seguía, precisamente, la necesidad de contar con estrategias pedagógicas sobre la enseñanza en clases multiculturales $(90,8 \%)$, apareciendo en tercer lugar la idea de que el motor de la formación sea el centro escolar (89,1\%). Las modalidades más extendidas en la práctica, conferencias y cursos impartidos por expertos, ocupaban el penúltimo lugar $(77,8 \%)$. 
TABLA 2

MODALIDADES DE FORMACIÓN DEL PROFESORADO EN EL ÁMBITO DE LA EI

\begin{tabular}{|c|c|c|c|c|c|c|c|}
\hline & \multicolumn{2}{|c|}{ Conformidad } & \multirow[b]{2}{*}{ 苋 } & \multicolumn{2}{|c|}{ Disconformidad } & \multirow[b]{2}{*}{ 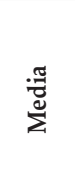 } & \multirow[b]{2}{*}{ 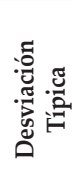 } \\
\hline & 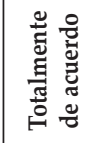 & 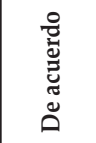 & & 本 & 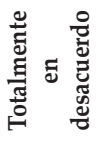 & & \\
\hline $\begin{array}{l}\text { a. Conferencias, cursos, impartidos por ex- } \\
\text { pertos }\end{array}$ & $21,6 \%$ & $56,3 \%$ & $15,2 \%$ & $4,7 \%$ & $2,3 \%$ & 2,10 & 870 \\
\hline $\begin{array}{l}\text { b. Conocimientos de las culturas minorita- } \\
\text { rias, mediante lecturas seleccionadas }\end{array}$ & $16,2 \%$ & $52,2 \%$ & $21,5 \%$ & $8,3 \%$ & $1,8 \%$ & 2,27 & 892 \\
\hline $\begin{array}{l}\text { c. Adquisición de estrategias pedagógicas } \\
\text { sobre la enseñanza en clases multiculturales }\end{array}$ & $37,8 \%$ & $53,1 \%$ & $6,1 \%$ & $1,4 \%$ & $1,7 \%$ & 1,76 & 767 \\
\hline $\begin{array}{l}\text { d. Conocimientos de otras experiencias exi- } \\
\text { tosas para gestionar la diversidad cultural }\end{array}$ & $38,4 \%$ & $52,9 \%$ & $5,3 \%$ & $1,9 \%$ & $1,4 \%$ & 1,75 & ,761 \\
\hline $\begin{array}{l}\text { e. Formación en el propio centro a partir } \\
\text { de los problemas que surgen al enseñar a } \\
\text { alumnos/as culturalmente diversos }\end{array}$ & $37,3 \%$ & $51,8 \%$ & $6,4 \%$ & $1,9 \%$ & $2,5 \%$ & 1,81 & 840 \\
\hline
\end{tabular}

TABLA 3

CONTENIDOS DE LA FORMACIÓN DEL PROFESORADO EN EL ÁMBITO DE LA EI

\begin{tabular}{|c|c|c|c|c|c|c|c|}
\hline & \multicolumn{2}{|c|}{ Conformidad } & \multirow[b]{2}{*}{ 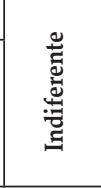 } & \multicolumn{2}{|c|}{ Disconformidad } & \multirow[b]{2}{*}{ 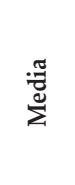 } & \multirow[b]{2}{*}{ 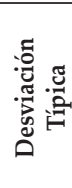 } \\
\hline & 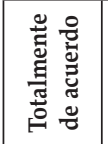 & 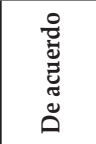 & & 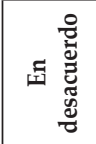 & 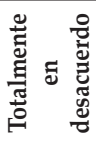 & & \\
\hline a. Conocimientos de otras lenguas & $9,8 \%$ & $40,8 \%$ & $30,1 \%$ & $15,2 \%$ & $4,2 \%$ & 2,63 & ,993 \\
\hline $\begin{array}{l}\text { b. La problemática social/legal/económica... } \\
\text { de los inmigrantes }\end{array}$ & $13,4 \%$ & $68,3 \%$ & $13,1 \%$ & $4,3 \%$ & $0,9 \%$ & 2,11 & ,710 \\
\hline c. Valores y socialización de la infancia & $19,8 \%$ & $67,2 \%$ & $9,2 \%$ & $2,6 \%$ & $1,1 \%$ & 1,98 & ,706 \\
\hline $\begin{array}{l}\text { d. Los estilos de aprendizaje y motivación } \\
\text { del alumnado inmigrante }\end{array}$ & $25,3 \%$ & $65,4 \%$ & $6,2 \%$ & $2,2 \%$ & $0,9 \%$ & 1,88 & ,683 \\
\hline $\begin{array}{l}\text { e. Comunicación e interacción en la familia } \\
\text { y en la sociedad }\end{array}$ & $21 \%$ & $68,2 \%$ & $8,2 \%$ & $1,7 \%$ & $0,9 \%$ & 1,93 & ,661 \\
\hline $\begin{array}{l}\text { f. Adquisición de estrategias pedagógicas } \\
\text { sobre negociación de conflictos en el aula }\end{array}$ & $33,4 \%$ & $56,7 \%$ & $6,5 \%$ & $1,7 \%$ & $1,7 \%$ & 1,81 & ,765 \\
\hline g. Aprendizaje cooperativo & $32,1 \%$ & $58,7 \%$ & $6,6 \%$ & $1,2 \%$ & $1,4 \%$ & 1,81 & ,728 \\
\hline
\end{tabular}

En relación con las valoraciones formuladas acerca de la pertinencia de incluir una serie de contenidos básicos para la formación en EI, destacó el elevado nivel de aceptación que recibe el aprendizaje cooperativo (90,8\%), seguido de cerca por la adquisición de estrategias pedagógicas sobre negociación de conflictos en el aula $(90,2 \%)$, los estilos de aprendizaje y motivación del alumnado inmigrante $(90,7 \%)$, la 
comunicación e interacción en la familia y en la sociedad $(89,2 \%)$ o los valores y la socialización de la infancia $(87,1 \%)$, en tanto que el conocimiento de otras lenguas, aún obteniendo una estimación positiva (50,6\%), figuraba en el último lugar de las preferencias.

\section{Influencia de algunas variables en la formación del colectivo docente en diversidad cultural}

Una vez completado el análisis descriptivo, pasamos a estudiar, utilizando técnicas de significación estadística, la influencia de determinadas características personales y profesionales de los encuestados/as en sus respuestas, tomando en consideración una decena de variables de estudio: edad, sexo, titulación académica, situación profesional, experiencia docente general, experiencia laboral con inmigrantes, interacción con alumnado inmigrante en ese curso escolar o en el anterior, desempeño de algún cargo directivo, etapa en la que se imparte docencia y participación en acciones formativas sobre EI.

Podemos afirmar que la edad y, en menor medida, la experiencia docente general y también la situación profesional, son las variables en torno a las cuales se presentaron las mayores diferencias significativas entre las respectivas valoraciones docentes. Por lo que respecta a la edad, la concentración más elevada de diferencias significativas se reflejó fundamentalmente entre el grupo de profesionales mayores de 50 años y los menores de 30 años. Los encuestados de mayor edad se mostraron menos escépticos sobre el nivel de preparación del profesorado, declarando poco entusiasmo hacia la inclusión de la EI (formación inicial y permanente) y menor consideración del concurso de mediadores interculturales en los centros con un número notable de alumnos inmigrantes. Paralelamente, el aumento en la edad de los encuestados disminuyó el nivel de conformidad ${ }^{3}$ o intensidad del acuerdo ${ }^{4}$ en su valoración de buena parte de las diferentes modalidades y contenidos propuestos para la EI.

En función de la experiencia docente general se observaron diferencias significativas en prácticamente los mismos ítems señalados con respecto a la variable edad, poniendo de relieve la estrecha correlación entre ambas magnitudes. Las diferencias se hicieron más asiduas entre las respuestas de los profesionales con menos de cinco años de práctica y, fundamentalmente, en aquellos con más de treinta años de oficio. Los cambios de percepción aparejados al incremento de la experiencia laboral discurrieron, obviamente, en sentido paralelo a aquellos que, según examinamos anteriormente, se producían al elevarse la edad de los encuestados.

La clasificación de los docentes en función de su diferente situación profesional puso en evidencia igualmente la existencia de ciertas regularidades de pensamiento entre los integrantes de determinados grupos. Como cabría esperar, se reproducen algunas de las diferencias observadas anteriormente al estudiar los cambios originados en virtud

3 El nivel de conformidad se obtiene como resultado de la suma de los porcentajes correspondientes a "Totalmente de acuerdo" y "De acuerdo". De igual manera, la disconformidad hace referencia al agrupamiento de "Totalmente en desacuerdo" y "En desacuerdo".

4 Disminución en los porcentajes de la columna "Totalmente de acuerdo" en favor de los correspondientes a "De acuerdo". 
TABLA 4

VARIACIONES SIGNIFICATIVAS EN LAS RESPUESTAS EN FUNCIÓN DE LA EDAD

\begin{tabular}{|c|c|c|c|c|c|}
\hline \multirow[b]{2}{*}{ Edad } & \multirow[b]{2}{*}{$\begin{array}{l}<30 \\
\text { años }\end{array}$} & \multirow[b]{2}{*}{$\begin{array}{l}>50 \\
\text { años }\end{array}$} & & \multicolumn{2}{|c|}{ ANOVA } \\
\hline & & & & Sig. & $\begin{array}{l}\text { Diferencia } \\
\text { de medias }\end{array}$ \\
\hline $\begin{array}{l}\text { Los profesores/as, en general, estamos prepara- } \\
\text { dos para responder a los desafíos de la Educación } \\
\text { Intercultural }\end{array}$ & $5,9 \%$ & $30,7 \%$ & \multirow{9}{*}{ 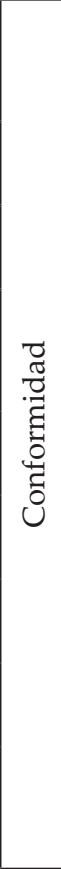 } & ,001 &, 598 \\
\hline $\begin{array}{l}\text { En la formación continuada del profesorado este tema } \\
\text { no está suficientemente atendido }\end{array}$ & $75,8 \%$ & $58 \%$ & & ,031 &,- 511 \\
\hline $\begin{array}{l}\text { La formación inicial del profesorado tiene que incluir } \\
\text { Educación Intercultural }\end{array}$ & $94,1 \%$ & $76,3 \%$ & & ,000 &,- 756 \\
\hline $\begin{array}{l}\text { Sería positivo que en centros con un número notable } \\
\text { de alumnos/as inmigrantes hubiera mediadores/as } \\
\text { interculturales }\end{array}$ & $96,9 \%$ & $78,6 \%$ & & ,001 &,- 584 \\
\hline \multicolumn{3}{|c|}{$\begin{array}{l}\text { La formación del profesorado en este ámbito debe seguir estas } \\
\text { modalidades: }\end{array}$} & & & \\
\hline $\begin{array}{l}\text { Conocimientos de las culturas minoritarias, mediante } \\
\text { lecturas seleccionadas }\end{array}$ & $84,9 \%$ & $63,2 \%$ & & ,010 &,- 558 \\
\hline $\begin{array}{l}\text { Adquisición de estrategias pedagógicas sobre la ense- } \\
\text { ñanza en clases multiculturales }\end{array}$ & $97,5 \%$ & $85,8 \%$ & & ,000 &,- 654 \\
\hline $\begin{array}{l}\text { Conocimientos de otras experiencias exitosas para ges- } \\
\text { tionar la diversidad cultural }\end{array}$ & $100 \%$ & $86,5 \%$ & & ,000 &,- 647 \\
\hline $\begin{array}{l}\text { Formación en el propio centro a partir de los proble- } \\
\text { mas que surgen al enseñar a alumnos/as culturalmente } \\
\text { diversos }\end{array}$ & $100 \%$ & $81,6 \%$ & & ,000 &,- 801 \\
\hline \multicolumn{3}{|l|}{ Los contenidos de formación deberían ser: } & \multirow{6}{*}{ 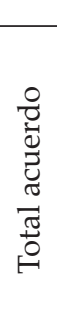 } & & \\
\hline Valores y socialización de la infancia & $35,3 \%$ & $13,9 \%$ & & ,005 &,- 319 \\
\hline $\begin{array}{l}\text { Los estilos de aprendizaje y motivación del alumnado } \\
\text { inmigrante }\end{array}$ & $47,1 \%$ & $13,5 \%$ & & ,000 &,- 509 \\
\hline Comunicación e interacción en la familia y en la sociedad & $41,2 \%$ & $15 \%$ & & , 000 &,- 403 \\
\hline $\begin{array}{l}\text { Adquisición de estrategias pedagógicas sobre negocia- } \\
\text { ción de conflictos en el aula }\end{array}$ & $52,9 \%$ & $26,4 \%$ & & ,005 &,- 432 \\
\hline Aprendizaje cooperativo & $55,9 \%$ & $20 \%$ & & 000 &,- 500 \\
\hline
\end{tabular}




\section{TABLA 5}

VARIACIONES SIGNIFICATIVAS EN LAS RESPUESTAS EN FUNCIÓN DE LA EXPERIENCIA DOCENTE

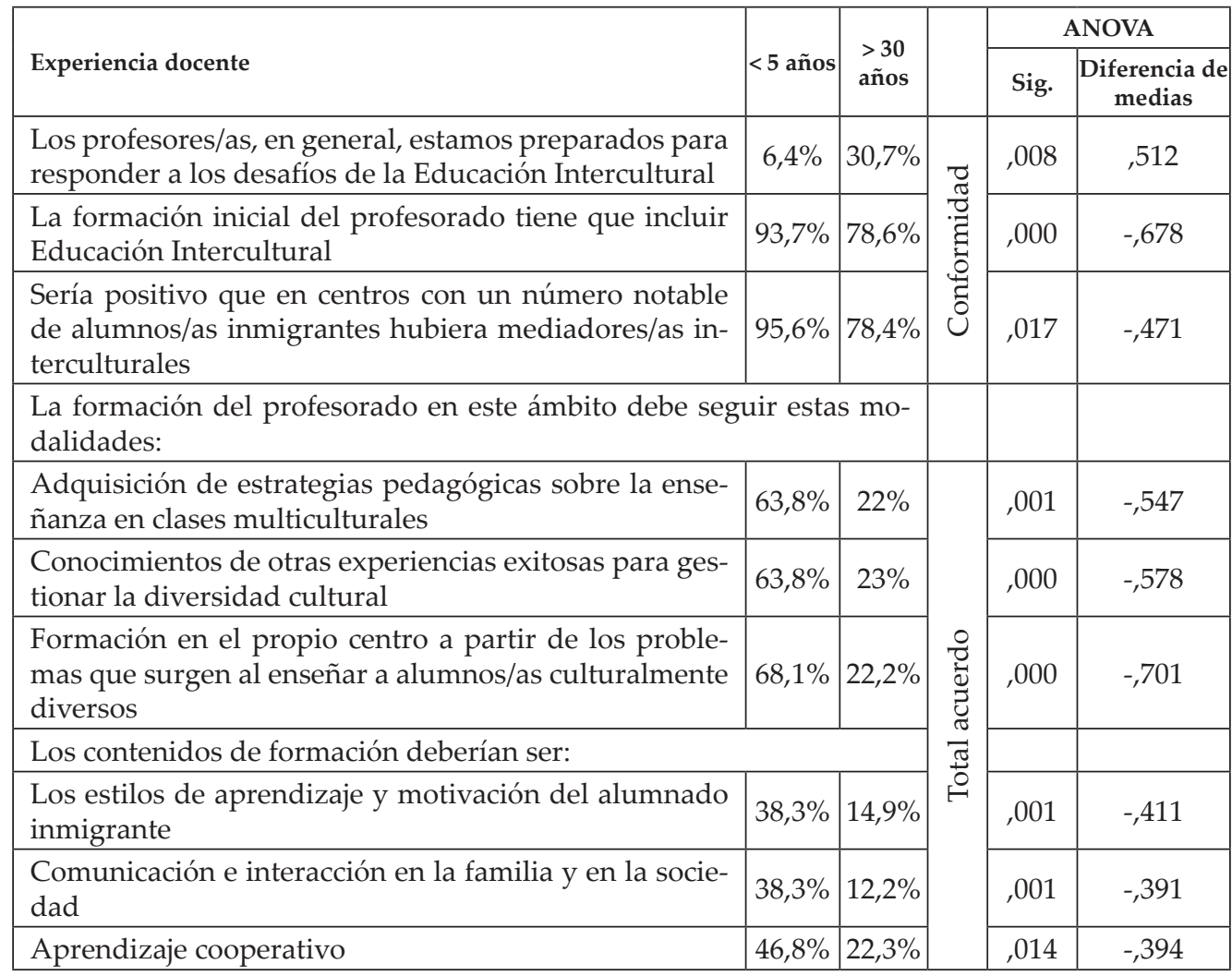

de las variables edad y experiencia docente, dado que los profesores en situación de 'propiedad provisional' suelen ser más jóvenes, en tanto aquellos que han alcanzado la 'propiedad definitiva' tienen, por lo regular, mayor edad y también más años de práctica profesional. 
TABLA 6

VARIACIONES SIGNIFICATIVAS EN LAS RESPUESTAS EN FUNCIÓN DE LA SITUACIÓN PROFESIONAL

\begin{tabular}{|c|c|c|c|c|c|}
\hline \multirow[b]{2}{*}{ Situación profesional } & \multirow[b]{2}{*}{ PD } & \multirow[b]{2}{*}{ PP } & & \multicolumn{2}{|c|}{ ANOVA } \\
\hline & & & & Sig. & $\begin{array}{l}\text { Diferencia } \\
\text { de medias }\end{array}$ \\
\hline $\begin{array}{l}\text { La formación inicial del profesorado tiene que incluir } \\
\text { Educación Intercultural }\end{array}$ & $24,8 \%$ & $53,1 \%$ & \multirow{6}{*}{$\begin{array}{l}0 \\
0 \\
0 \\
0 \\
0 \\
\tilde{0} \\
\tilde{\pi} \\
0 \\
0\end{array}$} & ,000 & ,485 \\
\hline \multicolumn{3}{|c|}{$\begin{array}{c}\text { La formación del profesorado en este ámbito debe seguir estas } \\
\text { modalidades: }\end{array}$} & & & \\
\hline $\begin{array}{l}\text { Conocimientos de otras experiencias exitosas para } \\
\text { gestionar la diversidad cultural }\end{array}$ & $34,4 \%$ & $53,1 \%$ & & , 010 & ,348 \\
\hline $\begin{array}{l}\text { Formación en el propio centro a partir de los } \\
\text { problemas que surgen al enseñar a alumnos/as } \\
\text { culturalmente diversos }\end{array}$ & $32,6 \%$ & $51,0 \%$ & & ,002 & ,399 \\
\hline \multicolumn{3}{|l|}{ Los contenidos de formación deberían ser: } & & & \\
\hline $\begin{array}{l}\text { Comunicación e interacción en la familia y en la } \\
\text { sociedad }\end{array}$ & $15,5 \%$ & $36,7 \%$ & & ,001 & ,297 \\
\hline
\end{tabular}

Nota. PD: Propiedad Definitiva. PP: Propiedad Provisional

Se observaron diferencias significativas en la variable género en algunos apartados objeto de estudio. Las profesoras reflejaron menor convencimiento que los maestros de poseer una preparación adecuada en EI. Además mostraron un nivel superior al reconocer la necesidad de disponer de mediadores interculturales, así como al valorar algunas modalidades de formación propuestas (conferencias y/o cursos impartidos por expertos y conocimiento de las culturas minoritarias mediante lecturas seleccionadas).

TABLA 7

VARIACIONES SIGNIFICATIVAS EN LAS RESPUESTAS EN FUNCIÓN DEL GÉNERO

\begin{tabular}{|c|c|c|c|c|c|}
\hline \multirow{2}{*}{ Género } & \multirow{2}{*}{ Femenino } & \multirow{2}{*}{ Masculino } & & \multicolumn{2}{|c|}{ Student } \\
\hline & & & & $t$ & $\mathrm{p} \leq$ \\
\hline $\begin{array}{l}\text { Considero que mi formación en Educación } \\
\text { Intercultural no es adecuada }\end{array}$ & $48,7 \%$ & $36,1 \%$ & \multirow{5}{*}{ 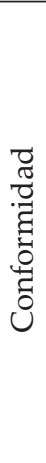 } & $-2,078$ & ,039 \\
\hline $\begin{array}{l}\text { Sería positivo que en centros con un número nota- } \\
\text { ble de alumnos inmigrantes hubiese mediadores } \\
\text { interculturales }\end{array}$ & $87,5 \%$ & $75 \%$ & & $-2,804$ & , 006 \\
\hline \multicolumn{3}{|c|}{$\begin{array}{l}\text { La formación del profesorado en este ámbito debe seguir estas modali- } \\
\text { dades: }\end{array}$} & & & \\
\hline Conferencias, cursos, impartidos por expertos & $80,6 \%$ & $69,6 \%$ & & $-2,011$ & , 046 \\
\hline $\begin{array}{l}\text { Conocimientos de las culturas minoritarias, me- } \\
\text { diante lecturas seleccionadas }\end{array}$ & $73,7 \%$ & $52 \%$ & & $-2,842$ & , 005 \\
\hline
\end{tabular}


La experiencia docente previa con alumnado de procedencia inmigrante apenas tuvo influencia significativa sobre sus opiniones en relación con este tema, sin que las diferencias encontradas vayan mucho más allá de la lógica disminución del sentimiento de falta de preparación que proporciona el incremento de la familiaridad con el alumnado foráneo.

TABLA 8

VARIACIONES SIGNIFICATIVAS EN LAS RESPUESTAS EN FUNCIÓN DE LA EXPERIENCIA CON ALUMNADO INMIGRANTE

\begin{tabular}{|c|c|c|c|c|c|}
\hline \multirow[b]{2}{*}{ Experiencia con alumnado inmigrante } & \multirow[b]{2}{*}{ Ninguna } & \multirow{2}{*}{$\begin{array}{l}>6 \\
\text { años }\end{array}$} & & \multicolumn{2}{|c|}{ ANOVA } \\
\hline & & & & Sig. & $\begin{array}{l}\text { Diferencia } \\
\text { de medias }\end{array}$ \\
\hline $\begin{array}{l}\text { Considero que mi formación en educación in- } \\
\text { tercultural es adecuada }\end{array}$ & $61,7 \%$ & $27,1 \%$ & \multirow{2}{*}{ 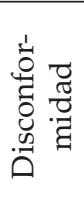 } & ,000 & ,941 \\
\hline $\begin{array}{l}\text { Los profesores/as, en general, estamos pre- } \\
\text { parados para responder a los desafíos de la } \\
\text { Educación Intercultural }\end{array}$ & $68,5 \%$ & $46,7 \%$ & & ,001 & 613 \\
\hline
\end{tabular}

Los profesionales que aseguraban haber participado en actividades formativas sobre la EI valoraron en mayor medida (como 'adecuada') su propia implicación en este campo. Además estimaron más necesaria la presencia de mediadores interculturales; también expresaron mayor grado de aprecio por la conveniencia de incluir la EI en la formación inicial del profesorado. Igualmente manifestaron un superior nivel de acuerdo con determinadas modalidades de formación en este terreno: conferencias y/o cursos impartidos por expertos, conocimiento de las culturas minoritarias mediante lecturas seleccionadas y adquisición de estrategias pedagógicas sobre la enseñanza en clases multiculturales.

TABLA 9

VARIACIONES SIGNIFICATIVAS EN LAS RESPUESTAS EN FUNCIÓN DE LA FORMACIÓN ESPECÍFICA EN EI

\begin{tabular}{|c|c|c|c|c|c|}
\hline \multirow{2}{*}{ Participación en actividades formativas } & \multirow{2}{*}{ Sí } & \multirow{2}{*}{ No } & & \multicolumn{2}{|c|}{ Student } \\
\hline & & & & $\mathbf{t}$ & $\mathrm{p} \leq$ \\
\hline $\begin{array}{l}\text { Considero que mi formación en educación intercultu- } \\
\text { ral es adecuada }\end{array}$ & $42,8 \%{ }^{5}$ & $22,5 \%$ & \multirow{7}{*}{ 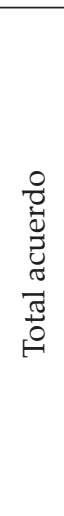 } & 4,804 & , 000 \\
\hline $\begin{array}{l}\text { Sería positivo que en centros con un número notable de } \\
\text { alumnos inmigrantes hubiese mediadores interculturales }\end{array}$ & $40,6 \%$ & $20,9 \%$ & & 2,681 & , 008 \\
\hline $\begin{array}{l}\text { La formación inicial del profesorado tiene que incluir } \\
\text { Educación Intercultural }\end{array}$ & $45,4 \%$ & $26 \%$ & & 2,569 & 011 \\
\hline \multicolumn{3}{|c|}{ La formación del profesorado en este ámbito debe seguir estas modalidades: } & & & \\
\hline Conferencias, cursos, impartidos por expertos & $33 \%$ & $16,9 \%$ & & 2,486 & ,014 \\
\hline $\begin{array}{l}\text { Conocimientos de las culturas minoritarias, mediante } \\
\text { lecturas seleccionadas }\end{array}$ & $24,5 \%$ & $12,8 \%$ & & 2,129 & ,035 \\
\hline $\begin{array}{l}\text { Adquisición de estrategias pedagógicas sobre la ense- } \\
\text { ñanza en clases multiculturales }\end{array}$ & $50,5 \%$ & $32,9 \%$ & & 2,070 & 040 \\
\hline
\end{tabular}

5 Para este ítem, los porcentajes corresponden a los encuestados que manifiestan conformidad. 
Se constató, por último, que otras variables, como la etapa en la que se imparte docencia, la titulación académica, el desempeño de algún cargo directivo e incluso la interacción en ese curso con alumnado de procedencia inmigrante, apenas tuvo impacto estadísticamente significativo en la orientación de las opiniones expresadas por el profesorado en relación con esta temática.

\section{CONCLUSIONES}

Entre las principales conclusiones que se derivan del análisis de las ideas del profesorado en el terreno de la EI, podemos destacar las siguientes:

- La necesidad de incluir la EI en el currículo de la formación del profesorado es algo generalmente aceptado.

- Las preferencias de los profesionales de la enseñanza por determinadas modalidades de formación en este campo difieren substantivamente del enfoque predominante en la Administración Educativa.

- Las opiniones expresadas dependen significativamente de la edad y experiencia docente de quien las formula.

La culminación lógica del análisis empírico que hemos podido realizar no es otra que hacer algunas sugerencias, y aún recomendaciones para diferentes esferas institucionales de actuación. En primer lugar, en virtud de la responsabilidad fundamental que corresponde a la Administración en este terreno, sería importante la creación de un Departamento específico de Orientación en EI. A este departamento le correspondería, entre otras funciones, la responsabilidad de elaborar y poner en marcha un plan de formación permanente, que incluyera entre sus ejes de desarrollo la asunción de la diversidad cultural como factor de provecho para la integración social de mayorías y minorías; así como el análisis del impacto que estas actividades de formación tienen en las aulas.

Deberían introducirse contenidos ligados al aprendizaje de la EI en los programas de formación inicial del profesorado; tratando de favorecer un mayor protagonismo de los docentes en el diseño de tales programas, incentivando su implicación en proyectos de investigación e innovación (cfr. Bennett, 2001).

El centro educativo debería ser el eje sobre el que se construya el plan de formación, por lo que habrá de disponer de los recursos humanos y materiales necesarios. Se debería potenciar en los centros el trabajo en equipo de los profesores, así como el establecimiento de cauces y mecanismos de contacto entre los profesionales de diferentes escuelas para posibilitar la creación de redes y la difusión eficiente de buenas prácticas.

Por último, necesitamos una mayor asociación estratégica entre la administración educativa y la misma universidad para definir los programas que convengan a la mejor articulación de conocimiento, disposición y competencia en EI por parte de los docentes. Hay muchas razones para reclamar esa confluencia en las políticas y, consiguientemente, en las decisiones. Pero una destaca sobremanera: la impostergable necesidad de ver en la formación del profesorado una de nuestras últimas oportunidades para mejorar la calidad del aprendizaje y de las competencias que niños y jóvenes precisan para su despliegue en un mundo global. 


\section{REFERENCIAS BIBLIOGRÁFICAS}

Aguado, M. T., Gil I. \& Mata, P. (2008). El enfoque intercultural en la formación del profesorado. Dilemas y propuestas. Revista Complutense de Educación, 19(2), 275-292.

Bennett, C. (2001). Genres of research in multicultural education. Review of Educational Research, 71(2), 171-217.

Colectivo IOÉ (1995). Extraños, distintos, iguales o las paradojas de la alteridad. Discurso de los españoles sobre los extranjeros. Revista de Educación, 307, 17-51.

Defensor del Pueblo (2003). Informe sobre la escolarización del alumnado de origen inmigrante en España: análisis descriptivo y estudio empírico (2 vols.) Madrid: Defensor del Pueblo. Recuperado el 30 de diciembre de 2007, de http://www.defensordelpueblo. es/informes2.asp.

Díaz-Aguado, M. J. (2003). Educación intercultural y aprendizaje cooperativo. Madrid: Pirámide.

Elliot, J. (1997). La investigación-acción en educación. Madrid: Ediciones Morata.

Escarbajal, A. (2010). Estudio de las respuestas socioeducativas ofrecidas a los inmigrantes en la región de Murcia. Revista de Investigación Educativa, 28(1), 157-170.

Essomba, M. A. (2006). Liderar escuelas interculturales e inclusivas: Equipos directivos y profesorado ante la diversidad cultural y la inmigración. Barcelona: Graó.

Esteve, J. M. (2004). La formación del profesorado para una educación intercultural. Bordón, 56(1), 95-115.

Fox, D. J. (1981). El proceso de investigación en educación. Pamplona: Ediciones Universidad de Navarra.

García Llamas, J. L. (2005). Educación intercultural. Análisis y propuestas. Revista de Educación, 336, 89-109.

Garreta, J. \& Llevot, N. (2003). El espejismo intercultural. La escuela de Cataluña ante la diversidad cultural. CIDE - Ministerio de Educación, Cultura y Deporte.

Giroux, H. (1997). Intelectuales públicos y la crisis de la enseñanza superior. Revista Interuniversitaria de Formación del Profesorado, 29, 77-87.

Grupo INTER (2006). Guía Inter. Una guía práctica para aplicar la educación intercultural en la escuela. Madrid: MEC

Jordán, J. A. (1994). La escuela multicultural: Un reto para el profesorado. Barcelona: Paidós. Jordán, J. A. (2004). La formación permanente del profesorado en educación intercultural. En AA.VV. La formación del profesorado en educación intercultural (pp. 11-48). MEC/Los Libros de la Catarata, Cuadernos de Educación Intercultural.

Leiva, J. (2010). Práctica de la interculturalidad desde la perspectiva docente: análisis y propuestas pedagógicas. Cultura y Educación, 22, 1, 67-84.

López Reillo, P. (2006). Una oportunidad para aprender. La dimensión intercultural en la formación del profesorado. Cabildo Insular de Tenerife, Área de Desarrollo Económico.

McAllister, G. \& Jordan, J. (2000). Cross cultural Competency and multicultural teacher education. Review of Educational Research, 70, 1, 3-24.

Marcelo, C. (1992). Desarrollo de la comprensión intercultural en los programas de formación inicial del profesorado. En X Congreso Nacional de Pedagogía (Ed.). Educación Intercultural en la perspectiva de la Europa Unida (Tomo II, pp. 501-535). Salamanca: Imprenta Provincial. 
MEC (2011). Ministerio de Educación y Ciencia. Enseñanzas no universitarias. Alumnado matriculado. Curso 2010-2011. Datos Avance. Recuperado el 21 de noviembre de 2011, de http://www.educacion.gob.es/dctm/ministerio/horizontales/estadisticas/ no-universitarias/alumnado/matriculado/2010-2011/5-rdos-al-ext-10pdf-alumnado. pdf?documentId=0901e72b80d1984b.

Nieto, S. \& Santos, M. A. (1997). Formación multi/intercultural del profesorado: perspectivas en los Estados Unidos y España. Teoría de la Educación. Revista Interuniversitaria, 9, 55-74.

Peñalva, A. \& Soriano, E. (2010). Objetivos y contenidos sobre interculturalidad en la formación inicial de educadores y educadoras. ESE. Estudios sobre Educación, 18, 37-57.

Pérez Gómez, A. I. (1992). La función y formación del profesor/a en la enseñanza para la comprensión. Diferentes perspectivas. En J. Gimeno \& A. Pérez Gómez (Eds.) Comprender y transformar la enseñanza (pp. 398-429). Madrid: Morata.

Santos, M. A. \& Lorenzo, M. (2003). Inmigración e acción educativa en Galicia. Vigo: Edicións Xerais de Galicia.

Santos, M. A.; Lorenzo, M. \& Priegue, D. (2011). Infancia de la inmigración y educación: la visión de las familias. Revista de Investigación Educativa, 29(1), 97-110.

Sercu, L. (2006). The foreign language and intercultural competence teacher: the acquisition of a new profesional identity. Intercultural Education, 17(1), 55-72.

Schön, D. A. (1998). El profesional reflexivo: cómo piensan los profesionales cuando actúan. Barcelona: Paidós.

Fecha de recepción: 06 de julio de 2012.

Fecha de revisión: 08 de julio de 2012.

Fecha de aceptación: 15 de enero de 2013. 